\title{
Leptoproduction of Neutrino Pairs in the Nuclear Coulomb Field.
}

\author{
A. Pich and J. Bernabéu
}

Departament de Física Teòrica, Universitat de València - València, Spain

(ricevuto il 9 Aprile 1985)

\begin{abstract}
Summary. - The cross-section for production of $\nu-\bar{\nu}$ pairs by highenergy leptons in the nuclear Coulomb field is calculated in the standard theory, allowing for arbitrary polarization of the incoming lepton beam. The differential distributions of the outgoing charged lepton are studied, showing that the scattered lepton peaks at low energies and emerges at appreciable angles. Incoherent contributions to the cross-section are also discussed.

PACS. 13.60. - Photon and charged-lepton interactions with hadrons.
\end{abstract}

\section{1. - Introduction.}

The trident production of neutrino pairs by high-energy leptons in the nuclear Coulomb field has been studied in the last years as a possible way to look for the number of lepton generations $\left({ }^{1-3}\right)$. In general, the nuclear coherent process

$$
\ell^{-}\left(p_{1}\right)+{ }^{A} \mathbf{Z}(P) \rightarrow \mathfrak{l}^{-}\left(p_{2}\right)+\bar{v}\left(p_{3}\right)+\nu\left(p_{4}\right)+{ }^{A} \mathbf{Z}\left(P^{\prime}\right)
$$

is mediated by both neutral-current and charged-current weak interactions,

(1) WU-KI Tung: Z. Phys. O, 4, 307 (1980).

$\left({ }^{2}\right)$ A. Pich and J. Bernabeu: Z. Phys. $O$, 14, 21 (1982).

(3) V. Barger, W. Y. Kevng and R. J. N. Phillips: Phys. Rev. D, 25, 677 (1982). 
when $v$ refers to the doublet partner neutrino of the incoming lepton. Using the $V-A$ character of charged currents, it is immediate to prove the suppression, by lepton mass factors, of this contribution for right-handed helicity of the incoming negative lepton. Therefore, selecting lepton beams with the appropriate helicity, the process (1) is described by an incoherent sum of neutralcurrent exchanges for each of the neutrino species.

The total cross-section for right-handed incoming leptons has been studied recently $\left({ }^{2}\right)$, in the high-energy limit, obtaining analytical expressions for the virtual-photon mass distribution. Numerical estimates of the unpolarized cross-section have also been given, by using the equivalent photon approximation $\left({ }^{3}\right)$. This method seems to work quite well for incident muons, but it does not for electrons by as much as $70 \%\left({ }^{4}\right)$.

In this paper we perform a detailed study of the process (1) allowing for arbitrary polarization of the lepton beam. For left-handed helicity, the chargedcurrent contribution can be related to the neutral-current one by a Fierz transformation. Furthermore, aside of factorized constants, the neutral-current contribution comes out to be helicity independent, so the polarization dependence of the cross-section amounts to a global factor only. We calculate the differential leptonic distributions. Our results are similar to the ones predicted $\left(^{5}\right)$ by the $V-A$-with only charged currents-, showing that the scattered lepton peaks at low energies and emerges at appreciable angles. These features are of importance in order to be able to separate in an actual experiment our process from the enormous background.

Noncoherent nuclear contributions to the cross-section are also discussed. While the corrections induced by the quasi-elastic peak show to be of some relevance, other possible contributions result negligible.

\section{2. - Amplitudes.}

The lowest-order contributions to process (1) are given by the diagrams of fig. 1. Charged-current interactions (diagrams 3)-5)) are only present when the final neutrino is the doublet partner of the charged lepton, whereas the noutral-current ones (diagrams 1),2)) give the same contribution for each of the neutrino species.

We study the process in the high-energy limit $\left(E_{1} \gg m\right)$, and take the contact limit for the two weak currents. This is justified by the fact that the present and near-future muon beam energies are still too low for the manifestation of boson propagator effects. Diagram 5) is suppressed because it has an extra

(4) A. Pich and J. Bernabeu: J. Phys. G, 8, L65 (1982).

$\left.{ }^{5}\right)$ K. FuJrkawa: Ann. Phys. (N. Y.), 68, 102 (1971). 


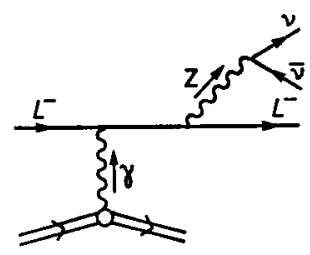

1)

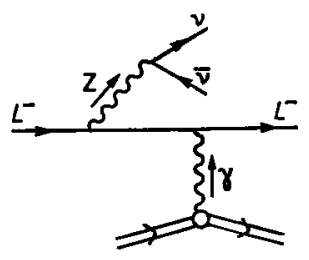

2)

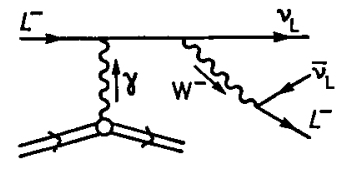

3)

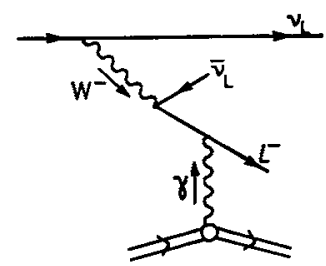

4)

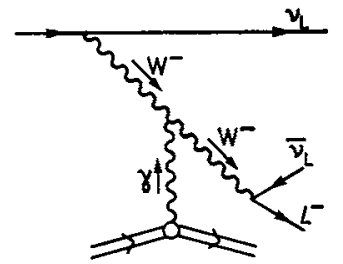

5)

Fig. 1. - Feynman diagrams contributing to leptoproduction of neutrino-antineutrino pairs in the nuclear Coulomb field.

heavy-boson propagator. The amplitudes are then given by

(2)

$$
\left\{\begin{array}{l}
T_{1}=\frac{Z e^{2} G}{2 \sqrt{2}} \frac{F\left(q^{2}\right)}{q^{2}}\left(P+P^{\prime}\right)^{\mu} N^{\sigma} \frac{L_{\sigma \mu}^{1}}{\left(p_{1}+q\right)^{2}-m^{2}}, \\
T_{2}=\frac{Z e^{2} G}{2 \sqrt{2}} \frac{F\left(q^{2}\right)}{q^{2}}\left(P+P^{\prime}\right)^{\mu} N^{\sigma} \frac{L_{\mu \sigma}^{2}}{\left(p_{2}-q\right)^{2}-m^{2}}, \\
T_{3}=\frac{Z e^{2} G}{\sqrt{2}} \frac{F\left(q^{2}\right)}{q^{2}}\left(P+P^{\prime}\right)^{\mu} \frac{L_{\sigma \mu}^{3} N_{3}^{\sigma}}{\left(p_{1}+q\right)^{2}-m^{2}}, \\
T_{4}=\frac{Z e^{2} G}{\sqrt{2}} \frac{F\left(q^{2}\right)}{q^{2}}\left(P+P^{\prime}\right)^{\mu} \frac{L_{\mu \sigma}^{4} N_{4}^{\sigma}}{\left(p_{2}-q\right)^{2}-m^{2}},
\end{array}\right.
$$

where the matrix elements of the leptonic amplitudes are

(3)

$$
\left\{\begin{array}{l}
L_{\sigma \mu}^{1}=\bar{u}\left(p_{2}\right) \gamma_{\sigma}\left(v-\gamma_{5}\right)\left(p_{1}+q\right) \gamma_{\mu} u\left(p_{1}\right), \\
L_{\mu \sigma}^{2}=\bar{u}\left(p_{2}\right) \gamma_{\mu}\left(p_{2}-q\right) \gamma_{\sigma}\left(v-\gamma_{5}\right) u\left(p_{1}\right), \\
N^{\sigma}=\bar{u}\left(p_{4}\right) \gamma^{\sigma}\left(1+\gamma_{5}\right) v\left(p_{3}\right), \\
L_{\sigma \mu}^{3}=\bar{u}\left(p_{4}\right) \gamma_{\sigma}\left(1+\gamma_{5}\right)\left(p_{1}+q\right) \gamma_{\mu} u\left(p_{1}\right), \\
L_{\mu \sigma}^{a}=\bar{u}\left(p_{2}\right) \gamma_{\mu}\left(p_{2}-q\right) \gamma_{\sigma}\left(1+\gamma_{5}\right) v\left(p_{3}\right), \\
N_{3}^{\sigma}=\bar{u}\left(p_{2}\right) \gamma^{\sigma}\left(1+\gamma_{5}\right) v\left(p_{3}\right), \\
N_{4}^{\sigma}=\bar{u}\left(p_{4}\right) \gamma^{\sigma}\left(1+\gamma_{5}\right) u\left(p_{1}\right) .
\end{array}\right.
$$


$q^{\mu} \equiv\left(P-P^{\prime}\right)^{\mu}$ is the virtual-photon momenta, $F\left(q^{2}\right)$ is the nuclear form factor and $v$ is the vector coupling of the $Z^{0}$ boson to the charged lepton. We have assumed the nucleus to be spinless in order to write the matrix element of the nuclear current.

With vertices of vector character there is conservation of helicity for $E_{1} \gg m$, so the charged currents are suppressed for right-handed helicity incoming leptons. For left-handed helicity, it is possible to relate the charged currents with the neutral currents by a Fierz transformation. We obtain (see appendix A)

$$
\left\{\begin{array}{l}
\left.\left.N_{3}^{\sigma} L_{\sigma \mu}^{3}\right|_{\lambda=-\frac{1}{2}} \simeq \frac{2}{1-v} N^{\sigma} L_{\sigma \mu}^{1}\right|_{\lambda=-\frac{1}{2}}, \\
\left.\left.N_{4}^{\sigma} L_{\mu \sigma}^{4}\right|_{\lambda=-\frac{1}{2}} \simeq \frac{2}{1-v} N^{\sigma} L_{\mu \sigma}^{2}\right|_{\lambda=-\frac{1}{2}} .
\end{array}\right.
$$

As a consequence, neutral-current and charged-current amplitudes are related by

$$
T_{\mathrm{ce}} \simeq \frac{4 \delta_{\lambda,-1} \frac{\delta_{l, \mathfrak{l}^{\prime}}}{1-v}}{1-v}
$$

where $\ell$ and $\ell^{\prime}$ refer to the incoming lepton and the charged partner of the outgoing neutrinos, respectively.

The neutral-current contribution to the cross-section, $T_{\mathrm{nc}}=T_{1}+T_{2}$, gives the following result:

$$
\left|T_{\mathrm{nc}}\right|^{2}=16(v+2 \lambda)^{2}\left(\frac{Z e^{2} G}{2 \sqrt{2}}\right)^{2} \frac{\left|F^{\prime}\left(q^{2}\right)\right|^{2}}{\left(q^{2}\right)^{2}}\left(P+P^{\prime}\right)^{\mu}\left(P+P^{\prime}\right)^{\nu} A^{\sigma \varrho} B_{\mu \nu \sigma \varrho}
$$

where the tensor $A^{\sigma o}$ arises from the product of neutrino currents, given by

$$
A^{\sigma \varrho}=p_{3}^{\sigma} p_{4}^{\varrho}+p_{3}^{\varrho} p_{4}^{\sigma}-g^{\alpha \varrho}\left(p_{3} \cdot p_{4}\right)+i \varepsilon^{\alpha \sigma \beta \varrho} p_{4 \alpha} p_{3 \beta}
$$

whereas $B_{\mu \nu \sigma_{e}}$ is a sum of three terms:

$$
\begin{aligned}
B_{\mu \nu \sigma \varrho}=\frac{B_{\mu \nu \sigma \varrho}^{(1)}}{\left(\left(p_{1}+q\right)^{2}-m^{2}\right)^{2}} & +\frac{B_{\mu \nu \sigma \varrho}^{(2)}}{\left(\left(p_{2}-q\right)^{2}-m^{2}\right)^{2}}+ \\
& +\frac{B_{\mu \nu \sigma \varrho}^{(3)}}{\left(\left(p_{1}+q\right)^{2}-m^{2}\right)\left(\left(p_{2}-q\right)^{2}-m^{2}\right)}
\end{aligned}
$$

obtained from the electromagnetic and neutral weak current vertices of the charged lepton. The three terms of eq. (8) are associated with the amplitudes $T_{1}, T_{2}$ and their interference. 


\section{3. - Cross-section.}

The cross-section of process (1) can be written as

$$
\begin{aligned}
\sigma\left(\mathfrak{l}^{-} \mathrm{Z} \rightarrow \mathfrak{l}^{-} \mathrm{Z} v_{l^{\prime}} \bar{\nu}_{l^{\prime}}\right)=C_{\lambda, l^{\prime}} & \frac{(Z \alpha G)^{2}}{2 M E_{1} \pi^{6}} \sin ^{4} \theta_{\mathrm{w}} \cdot \\
& \cdot \int \frac{\mathrm{d}^{3} P^{\prime}}{2 E^{\prime}} \frac{\left|E^{\prime}\left(q^{2}\right)\right|^{2}}{\left(q^{2}\right)^{2}}\left(P+P^{\prime}\right)^{\mu}\left(P+P^{\prime}\right)^{\nu} M_{\mu \nu}\left(p_{1}, q\right),
\end{aligned}
$$

where $M_{\mu \nu}\left(p_{1}, q\right)$ plays the role of the leptonic electromagnetic tensor

$$
M_{\mu \nu}\left(p_{1}, q\right) \equiv \int \frac{\mathrm{d}^{3} p_{2}}{2 E_{2}} \frac{\mathrm{d}^{3} p_{3}}{2 E_{3}} \frac{\mathrm{d}^{3} p_{4}}{2 E_{4}} \delta\left(p_{1}+q-p_{2}-p_{3}-p_{4}\right) A^{\sigma} \varrho B_{\mu \nu \sigma \varrho}
$$

and

$$
C_{\lambda, l^{\prime}}=\left(1-\delta_{\lambda,-\frac{1}{2}} \frac{1+2 \delta_{l, l^{\prime}}}{2 \sin ^{2} \theta_{\mathrm{w}}}\right)^{2}
$$

To proceed with the evaluation of $M_{\mu \nu}$, we introduce a set of invariant variables

$$
\left\{\begin{array}{lll}
x_{1}=-q^{2}, & x_{2}=\left(p_{1} \cdot q\right), & x_{3}=\left(p_{2} \cdot q\right), \\
x_{5}=\left(p_{1} \cdot p_{2}\right), & x_{\mathrm{B}}=\left(p_{1} \cdot p_{4}\right), & x_{7}=\left(p_{2} \cdot P\right)
\end{array}\right.
$$

in terms of which we write the invariant integrands.

Since the neutrino pair is not detected experimentally, it is convenient to start with the integration over the four-momenta $p_{3}$ and $p_{4}$. The only dependence on these variables is through the tensor $A^{\sigma e}$, and it is obvious that these integrations can only depend on the $Z^{0}$ four-momentum $k^{\mu}=\left(p_{1}+q-p_{2}\right)^{\mu}$, so we have

$$
I^{\sigma \varrho} \equiv \int \frac{\mathrm{d}^{3} p_{3}}{2 E_{3}} \frac{\mathrm{d}^{3} p_{4}}{2 E_{4}} \delta\left(p_{3}+p_{4}-k\right) A^{\sigma \varrho}=a_{1} g^{\sigma \varrho}+a_{2} k^{\sigma} k^{\varrho},
$$

where $a_{1}$ and $a_{2}$ are invariants. Note that $I^{\sigma e}$ is symmetric. The piece of $M_{\mu \nu}$ relevant in eq. (9) is its symmetric part. In the three terms of $B_{\mu \nu \sigma o}$, the symmetric part under $\mu \leftrightarrow \nu$ exchange has an helicity dependence which is antisymmetric under $\sigma \leftrightarrow \varrho$ exchange. Under contraction with $I^{\sigma Q}$, we conclude that the symmetric part of the electromagnetic tensor $M_{\mu v}$ becomes helicity independent. 
To evaluate the coefficients $a_{1,2}$ it is necessary to calculate only certain combinations of the $I^{\sigma e}$ components, i.e.

$$
\left\{\begin{array}{l}
a_{1}=\frac{1}{4} I_{\sigma}^{\sigma}-\frac{k^{2}}{4\left(p_{1} k\right)^{2}} p_{1 \sigma} p_{1 \varrho} I^{\sigma \varrho} \\
a_{2}=\frac{1}{\left(p_{1} k\right)^{2}} p_{1 \sigma} p_{1 \varrho} I^{\sigma} \varrho
\end{array}\right.
$$

The $p_{3}$ and $p_{4}$ integrations are easily computed in the centre-of-mass frame of the neutrino pair $\left(k=0, \hat{q}=\hat{z}, \hat{p}_{1} \in\right.$ plane $\left.(y=0)\right)$ and we obtain

$$
I^{\sigma e}=\frac{\pi}{6}\left(k^{\sigma} k^{\varrho}+\left(x_{1}-2\left(x_{2}-x_{3}-x_{5}\right) g^{\sigma e}\right)\right)
$$

The $k^{\sigma} k^{\varrho}$ term can be neglected when contracted with $B_{\mu \nu \sigma_{\varrho}}$, because it leads to a term proportional to the lepton mass. We then have

$$
M_{\mu \nu}\left(p_{1}, q\right) \simeq \frac{\pi}{6} \int \frac{\mathrm{d}^{3} p_{2}}{2 E_{2}}\left(x_{1}-2\left(x_{2}-x_{3}-x_{5}\right)\right) B_{\mu \nu \sigma^{\sigma}}
$$

As pointed out above, the relevant part of $\boldsymbol{M}_{\mu v}$ is helicity independent, so we recover the results of ref. $\left({ }^{2}\right)$ for the total cross-section and the virtual-photon mass distribution. We must only include the $C_{\lambda, \ell^{\prime}}$ factor of eq. (11) or, if we sum over all neutrino families, we just multiply by

$$
g(N, \lambda) \equiv \sum_{\ell^{\prime}} C_{\lambda, l^{\prime}}=(N-1)\left(1-\frac{\delta_{\lambda,-\frac{1}{2}}}{2 \sin ^{2} \theta_{\mathrm{w}}}\right)^{2}+\left(1-\frac{3 \delta_{\lambda,-\frac{1}{2}}}{2 \sin ^{2} \theta_{\mathrm{w}}}\right)^{2}
$$

where $N$ is the number of fermionic generations. For $\lambda=+\frac{1}{2}, g(N, \lambda)=N$ as it should. The cross-section is thus given by

$$
\begin{aligned}
\sigma\left(l^{-}+\mathrm{Z} \rightarrow \mathfrak{l}^{-}+\mathrm{Z}+\Sigma v \bar{v}\right)= & g(N, \lambda) \sigma_{0} Z^{2} E_{1} \cdot \\
& \cdot \int_{\underline{x}_{1}}^{\bar{x}_{1}} \frac{\mathrm{d} x_{1}}{\sqrt{x_{1}}}\left|F\left(x_{1}\right)\right|^{2}\left\{Z\left(x_{1}\right)+\ln \left(2 E_{1} \sqrt{x_{1}} / m^{2}\right)\right\},
\end{aligned}
$$

where

$$
\left\{\begin{array}{l}
Z\left(x_{1}\right)=-35 / 24-10 m^{2} / x_{1}-\left(1+2 m^{2} / x_{1}-20 m^{4} / x_{1}^{2}\right) \frac{1}{f} \ln \left(\frac{f+1}{f-1}\right) \\
f \equiv\left(1+4 m^{2} / x_{1}\right)^{\frac{1}{2}} \\
\sigma_{0} \equiv \frac{2 \alpha^{2} G^{2}}{27 \pi^{3}} \sin ^{4} \theta_{\mathrm{w}} .
\end{array}\right.
$$




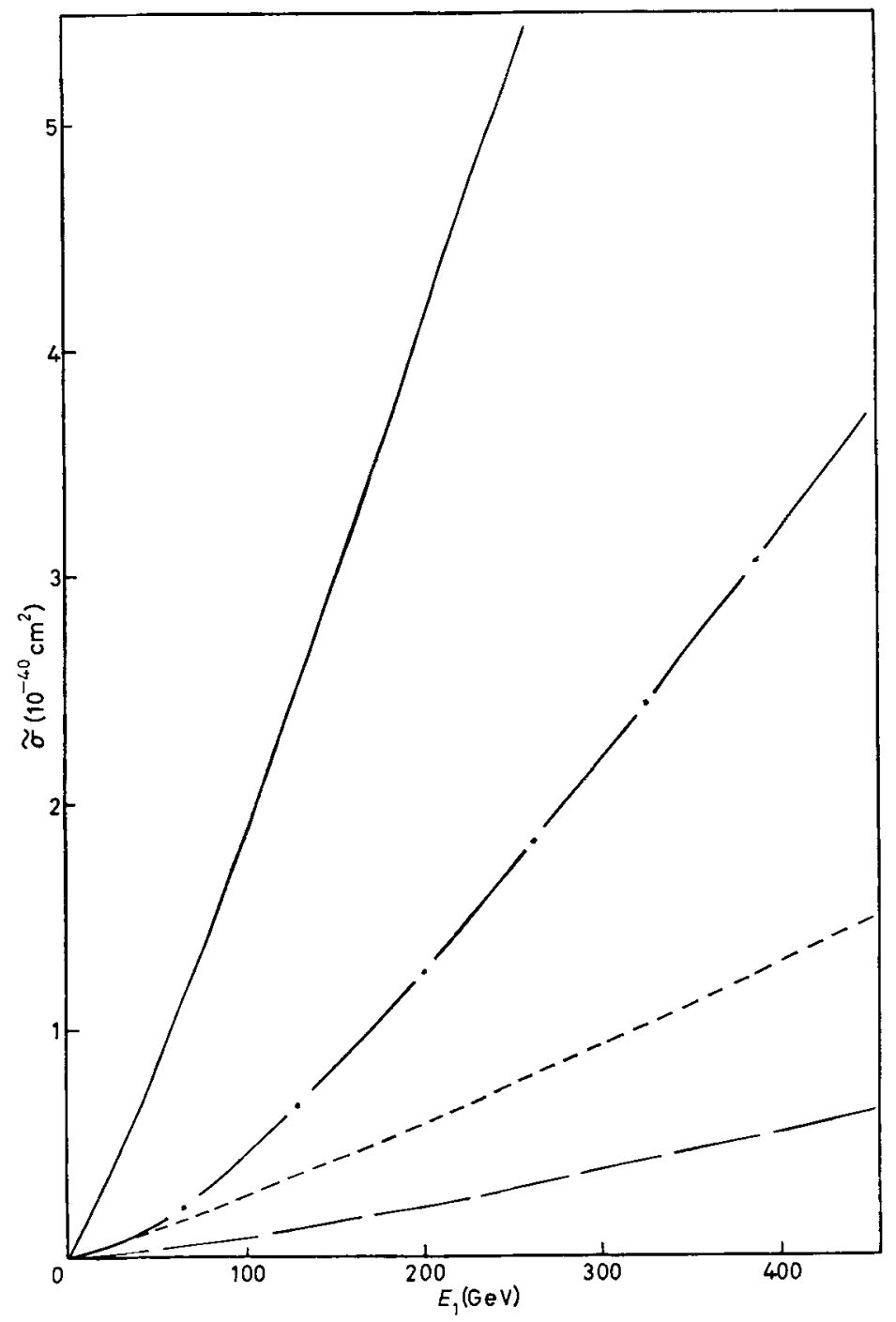

Fig. 2. - Normalized cross-section $(\tilde{\sigma} \equiv \sigma / g(N, \lambda))$ as a function of the incoming energy. The different curves correspond to electron over ${ }^{208} \mathrm{~Pb}\left(-\_\right)$, muon over ${ }^{208} \mathrm{~Pb}(-\cdot-\cdot-)$ electron over ${ }^{56} \mathrm{Fe}(---)$ and muon over ${ }^{56} \mathrm{Fe}(---)$.

The integrated cross-section has the following behaviour with the incoming energy:

$$
\sigma=g(N, \lambda) \sigma_{0} E_{1}\left(\alpha+\beta \ln \left(2 E_{1} / m\right)\right)
$$

where $\alpha, \beta$ depend on the target and on the incoming charged lepton. We have evaluated these cross-section coefficients using the experimental nuclear form 
factor $\left({ }^{6}\right)$ for ${ }^{56} \mathrm{Fe}$ and ${ }^{208} \mathrm{~Pb}$ and the current values of the weak interaction parameters ( ${ }^{7}$. The results are given in table $I$ for incoming electrons and muons.

TABLE I. - Parameters of the elastic $(\alpha, \beta)$, quasi-elastic $(\gamma, \eta)$ and total cross-section $(A, B)$, for the different cases we have considered. All values are given in $\mathrm{GeV}$.

\begin{tabular}{|c|c|c|c|c|c|c|c|}
\hline & & $\alpha$ & $\gamma$ & $A$ & $\bar{\beta}$ & $\eta$ & $B$ \\
\hline & ${ }^{56} \mathrm{Fe}$ & $-\quad 563.8$ & -76.7 & $-\quad 640.5$ & 106.8 & 18.2 & 125.0 \\
\hline & ${ }^{56} \mathrm{Fe}$ & -607.7 & -135.2 & $-\quad 742.9$ & 106.8 & 18.2 & 125.0 \\
\hline$m_{\mu}$ & ${ }^{208} \mathrm{~Pb}$ & -3947 & -242 & -4189 & 692.6 & 57.4 & 750.0 \\
\hline$m_{\mathrm{e}}$ & ${ }^{208} \mathrm{~Pb}$ & -3698 & -426 & -4125 & 692.6 & 57.4 & 750.0 \\
\hline
\end{tabular}

In fig. 2 we plot the ratio $\tilde{\sigma} \equiv \sigma / g(N, \lambda)$ for the resulting cross-sections as functions of the incoming energy. The polarization effects are described entirely by the factor $g(N, \lambda)$. For three generations, we have

$$
g\left(3, \frac{1}{2}\right)=3, \quad g\left(3,-\frac{1}{2}\right)=33.25, \quad \bar{g}(3)=18.12 .
$$

We conclude that, if the number of generations is not very large, the chargedcurrent contribution is dominant for left-handed helicity. For unpolarized muons at $300 \mathrm{GeV}$, and assuming three generations, the cross-section is 6.6 $\cdot 10^{-40} \mathrm{~cm}^{2}$ for ${ }^{56} \mathrm{Fe}$ and $4 \cdot 10^{-39} \mathrm{~cm}^{2}$ for ${ }^{208} \mathrm{~Pb}$.

\section{4. - Energy spectrum.}

To extract the differential distributions of the outgoing charged lepton, we have to change the strategy based on the global properties of the electromagnetic leptonic tensor $M_{\mu \nu}$. To proceed with the differential distributions, we start from eq. (16), inserted in eq. (9).

We perform the $p_{2}$ integration in the laboratory frame, but choosing an special orientation for the axis: $\boldsymbol{P}=\mathbf{0},\left(\boldsymbol{p}_{\mathbf{1}}+\boldsymbol{q}\right) \| \hat{z}, \boldsymbol{q} \in$ plane $(y=0)$. It is convenient to change the variable $x_{5}$ by the new one $z=x_{3}+x_{5}$. One obtains

$$
\left\{\begin{array}{l}
\frac{\mathrm{d}^{3} p_{2}}{2 E_{2}}=\frac{\pi}{\sqrt{W}} \mathrm{~d} E_{2} \mathrm{~d} z \frac{\mathrm{d} \varphi_{2}}{2 \pi} \\
W=\left(q^{0}+E_{1}\right)^{2}-2 x_{2}+x_{1}-m^{2}
\end{array}\right.
$$

$\left({ }^{6}\right)$ G. Key, H. Frank, W. Schütz and H. Theissen: Z. Phys., 265, 401 (1973); J. Heisenberg, R. Hofstadter, J. C. McCarthy, I. Sick, B. C. Clark, R. Herman and D. G. Ravenhall: Phys. Rev. Lett., 23, 1402 (1969).

(7) I. LIEDE and M. Roos: Nucl. Phys. B, 167, 397 (1980); J. E. Kim, P. LaNGacker, M. Levine and H. H. Williams: Rev. Mod. Phys., 53, 211 (1981). 
In this frame, only $x_{3}$ depends on the azimuth $\varphi_{2}$, so the $\varphi_{2}$ integration is immediate.

The $P^{\prime}$ integration is also made in the laboratory frame, but with the ordinary axis orientation, i.e. $\boldsymbol{P}=\mathbf{0}, \boldsymbol{p}_{\mathbf{1}} \| \hat{z}$. We have

$$
\frac{\mathrm{d}^{3} P^{\prime}}{2 E^{\prime}}=\frac{\pi}{2 M\left|\boldsymbol{p}_{1}\right|} \mathrm{d} x_{1} \mathrm{~d} x_{2} .
$$

To obtain the phase-space limits is a very tedious process, and it is outlined briefly in appendix $\mathrm{B}$, where the integration limits for $x_{1}, x_{2}, E_{2}$ and $z$ are given. The kinematical upper limit for $x_{1}$ is not effective, because the presence of the nuclear form factor in the virtual-photon mass distribution limits $x_{1}$ to small values.

It is possible to get an analytic estimate of the high-energy tail of the energy spectrum for zero-mass leptons. In this case, the integration limits reduce to

$$
\underline{z} \simeq x_{2} E_{2} / E_{1}, \quad \bar{z} \simeq x_{2}, \quad \underline{x}_{2} \simeq \frac{1}{2} x_{1}, \quad \bar{x}_{2} \simeq E_{1} \sqrt{x_{1}}
$$

so, for $E_{2} \gg m,\left\langle x_{1}\right\rangle^{\frac{1}{2}}$, we obtain

$$
\frac{\mathrm{d} \sigma}{\mathrm{d} E_{2}}(\lambda) \simeq g(N, \lambda) \sigma_{0} \beta\left(E_{1} / E_{2}-1\right)\left(1-E_{2}^{3} / E_{1}^{3}\right)
$$

where $\beta$ is the coefficient of the logarithmic term in eq. (20).

The energy distribution is strongly peaked at low energies, where the estimate (25) is not valid. We have evaluated the spectrum numerically, for incoming muons and electrons on ${ }^{56} \mathrm{Fe}$. The resulting distribution is plotted in fig. 3 for incoming energies of 100 and $300 \mathrm{GeV}$. We have drawn the function $\tilde{\sigma}\left(E_{2}\right) \equiv\left(\mathrm{d} \sigma / \mathrm{d} E_{2}\right)(\lambda) / g(N, \lambda)$. For outgoing energies larger than $1 \mathrm{GeV}$, we find a similar behaviour for muons and electrons, though with slightly larger values in the latter case. In this range of energies, eq. (25) is a good approximation. One sees that the maximum value of the distribution appears very close to the lower limit of $E_{2}, \tilde{\sigma}\left(E_{2}\right)$ falling rapidly to zero for $E_{2}=m$. For the two incoming energies considered, the maximum for $\tilde{\sigma}\left(E_{2}\right)$ occurs at values of $E_{2}$ around $600 \mathrm{MeV}$ in the muon case and $3 \mathrm{MeV}$ in the electron case.

The mean values for $E_{2}$ are given in table II. One notices that the charged

TABLE II. - Mean values of the outgoing energy $E_{2}$ and the dispersion angle $\theta_{2}$, for incoming energies of 100 and $300 \mathrm{GeV}$.

\begin{tabular}{lllll}
\hline & $\left\langle E_{2}\right\rangle(\mathrm{GeV})$ & & $\left\langle\theta_{2}\right\rangle$ (degrees) \\
\hline$E_{1}(\mathrm{GeV})$ & muon & electron & muon & electron \\
100 & 12.7 & 6.2 & 3.0 & 13.8 \\
300 & 30.7 & 15.6 & 2.9 & 10.7 \\
\hline
\end{tabular}




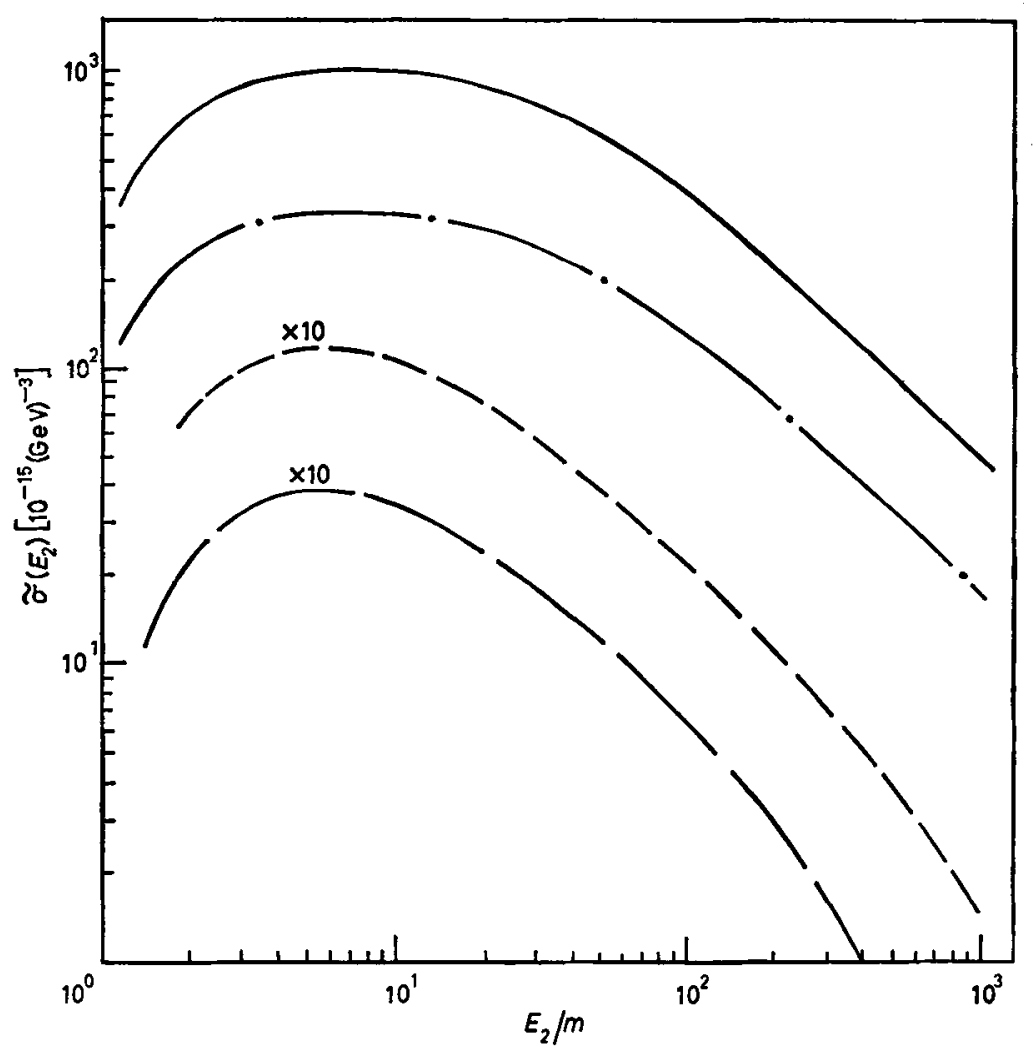

Fig. 3. - Energy distribution of the final charged lepton. The values of $\tilde{\sigma}\left(E_{2}\right)$ are given in $10^{-15}(\mathrm{GeV})^{-3}$ for electrons of $100(-\cdot-\cdot-)$ and $300(-) \mathrm{GeV}$, and $10^{-16}(\mathrm{GeV})^{-3}$ for muons of $100(---)$ and $300(--) \mathrm{GeV}$.

lepton losts a big amount of its energy during the neutrino-antineutrino radiation process. This fact helps for the detection of the process through the low-energy outgoing charged lepton, because the main background mainly produces energetic charged leptons.

\section{5. - Angular distribution.}

To obtain the angular distribution of the final charged lepton we work in the laboratory frame, referring the $\hat{p}_{2}$-direction to the axis defined by the incoming lepton, i.e. we choose $\boldsymbol{P}=\mathbf{0}, \boldsymbol{p}_{1} \| \hat{z}$. Taking into account the cylinder symmetry of the collision process, we have

$$
\frac{\mathrm{d}^{3} p_{2}}{2 E_{2}} \rightarrow \pi\left|\boldsymbol{p}_{2}\right| \mathrm{d} E_{2} \mathrm{~d}\left(\cos \theta_{2}\right) .
$$


The angular dependences come from the invariant $x_{5}$, given by

$$
x_{5}=E_{1} E_{2}-\left|\boldsymbol{p}_{1}\right|\left|\boldsymbol{p}_{2}\right| \cos \theta_{2} .
$$

To perform the $\boldsymbol{P}^{\prime}$-integration we take a different axis orientation, choosing the frame $\boldsymbol{P}=\mathbf{0},\left(\boldsymbol{p}_{1}-\boldsymbol{p}_{2}\right) \| \hat{z}, \boldsymbol{p}_{2} \in$ plane $(y=0)$. It is convenient to substitute $x_{2}$ by the now variable $y=x_{2}-x_{3}$, so

$$
\frac{\mathrm{d}^{3} P^{\prime}}{2 E^{\prime}}=\frac{\pi}{2 M \sqrt{H}} \mathrm{~d} x_{1} \mathrm{~d} y \frac{\mathrm{d} \varphi_{a}}{2 \pi},
$$

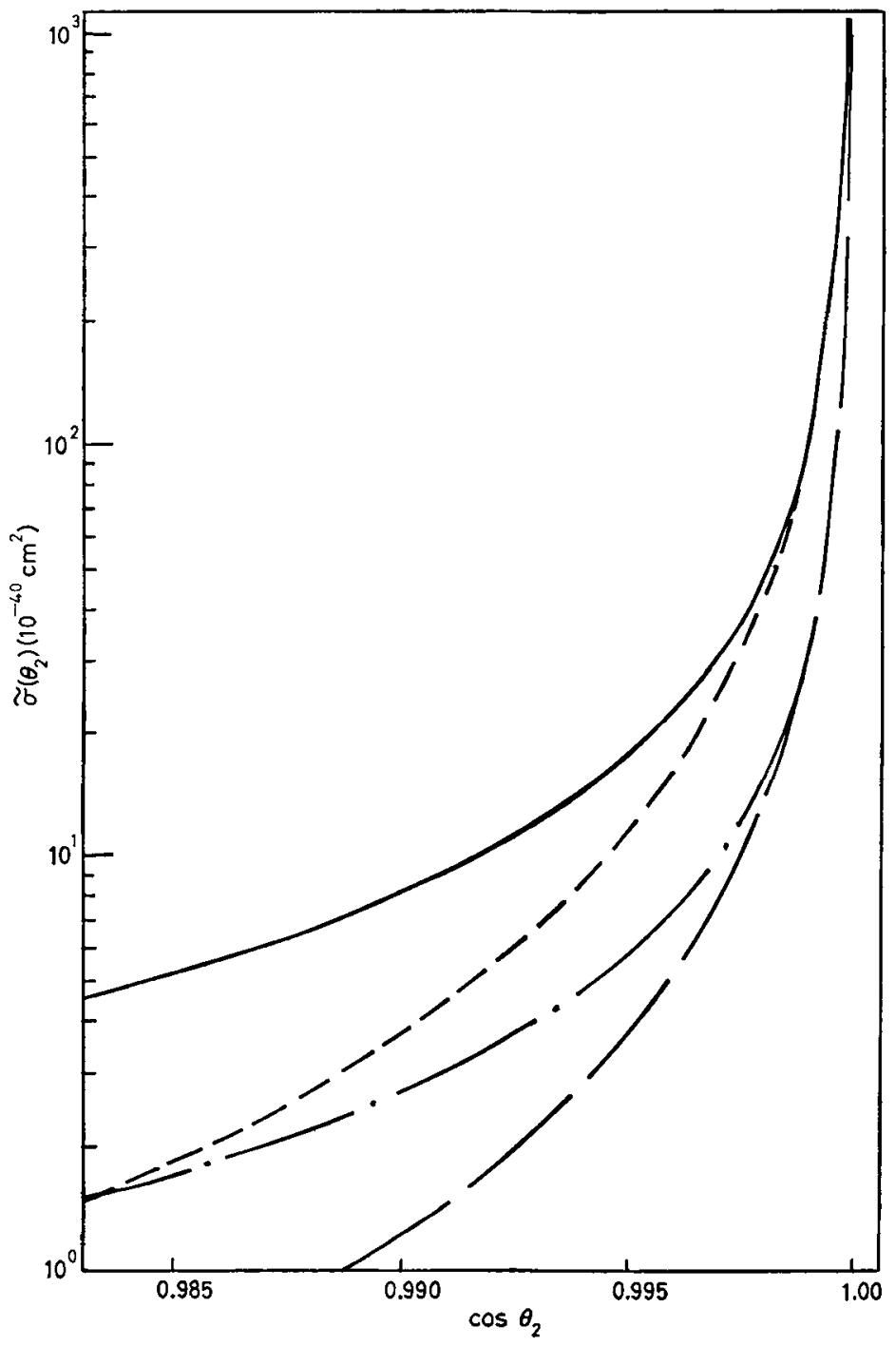

Fig. 4. - Angular distribution of the final charged lepton for electrons of $100(-\cdot-\cdot-)$ and $300(-) \mathrm{GeV}$ and muons of $100(--)$ and $300(--) \mathrm{GeV}$. 
where $H=\left(E_{1}-E_{2}\right)^{2}+2\left(x_{5}-m^{2}\right)$. The only $\varphi_{q}$-dependence comes through $x_{3}$ and this integration can be made analytically. The phase-space limits are given in appendix 0 .

By performing numerically the integrations over $x_{1}, E_{2}$ and $y$, we have studied the angular distribution behaviour for incoming muons and electrons on ${ }^{56} \mathrm{Fe}$, at incident energies of 100 and $300 \mathrm{GeV}$. The results are given in fig. 4, showing the quantity $\tilde{\sigma}\left(\theta_{2}\right) \equiv\left(\mathrm{d} \sigma / \mathrm{d}\left(\cos \theta_{2}\right)\right)(\lambda) / g(N, \lambda)$. As expected, the angular distribution is strongly peaked in the forward direction. Nevertheless, one obtains appreciable mean values for the dispersion angle, as shown in table II.

\section{Quasi-elastic contribution.}

The results discussed up to now come from the assumed dominance of the coherent nuclear process. There are additional contributions coming from the nuclear excitations induced by the virtual photon. We discuss here the corrections due to the quasi-elastic peak contribution. This corresponds to the incoherent interaction of the virtual photon with the individual nucleons. One needs in the hadronic vertex the matrix element of the electromagnetic current for a spin- $\frac{1}{2}$ particle

$$
\left\langle J_{\mu}\right\rangle=e \bar{u}\left(P^{\prime}\right)\left(\gamma_{\mu} F_{1}\left(q^{2}\right)-i \frac{\sigma_{\mu \nu} q^{\nu}}{2 M} F_{2}\left(q^{2}\right)\right) u(P),
$$

where the notation is conventional and $M$ is the nucleon mass. The dominant contributions come from the virtual photons with $x_{1} \sim 0.1(\mathrm{GeV})^{2}$, so the $x_{1} \ll x_{i}(i \geqslant 2)$ approximation, used in the calculation of the leptonic tensor, remains valid. On the other hand, one cannot neglect any longer the virtualphoton energy $q^{0}=-x_{1} / 2 M$. We have used the dipole fit for the Sachs form factors $\left(^{8}\right)$ of the nucleon and the results for the virtual-photon mass distribution are given in fig. 5 , as

$$
x_{1} \tilde{\sigma}_{n}\left(x_{1}\right) \equiv x_{1} \frac{\mathrm{d} \sigma_{n}}{\mathrm{~d} x_{1}} / g(N, \lambda)
$$

where the subindex $n$ refers to the nucleon considered, either proton or neutron. As seen, the neutron contribution is much smaller than the proton one and we can safely neglect it.

The contribution of the nuclear quasi-elastic peak to the cross-section of the process is then

$$
\sigma_{\mathrm{qe}} \simeq Z \sigma_{\mathrm{p}} \simeq g(N, \hat{\lambda}) \sigma_{0} E_{1}\left(\gamma+\eta \ln \left(2 E_{1} / m\right)\right)
$$

(8) M. Gourdin: Phys. Rep., 11, 29 (1974). 


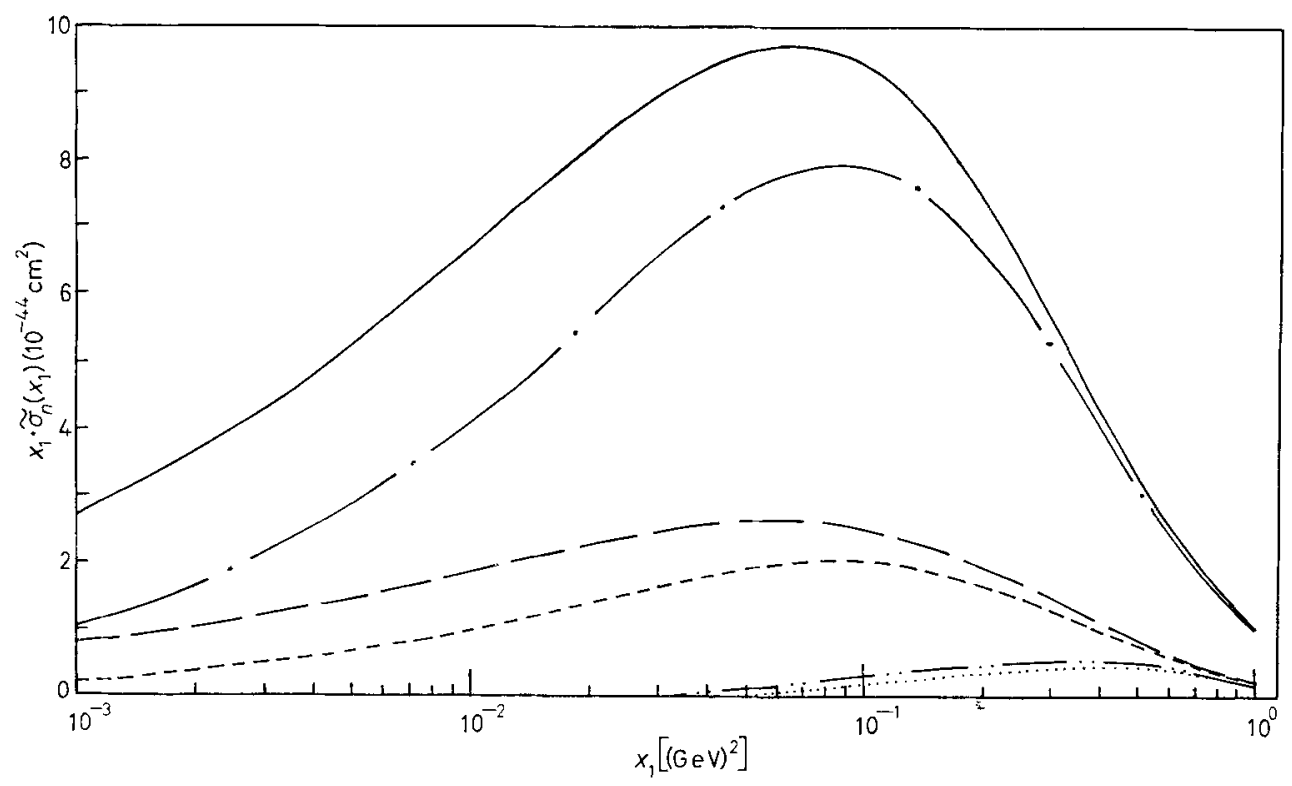

Fig. 5. - Virtual-photon mass distribution of the quasi-elastic process. The different curves correspond to incoming electrons of $100(---)$ and $300(-) \mathrm{GeV}$ over a proton, incoming muons of $100(--)$ and $300(-\cdot-\cdot-) \mathrm{GeV}$ over a proton, and incoming electrons $(-\cdots-\cdots-)$ and muons $(\cdots \cdots)$ of $300 \mathrm{GeV}$ over a neutron.

The values of the coefficients $\gamma, \eta$ are given in table I and when compared with eq. (20), the sum of coherent and incoherent cross-sections has the same dependence with the incoming energy, with the substitution $\alpha \rightarrow A=\alpha+\gamma$, $\beta \rightarrow B=\beta+\eta$.

As expected on physical grounds, the modification introduced by the quasielastic contribution is more important for ${ }^{56} \mathrm{Fe}$ than for ${ }^{208} \mathrm{~Pb}$. It is somewhat more relevant in the muonic case than in the electronic one. At incoming energies of $300 \mathrm{GeV}$, the quasi-elastic cross-section represents $18 \%$ for muons and iron, $12 \%$ for electrons and iron, $11 \%$ for muons and lead and $6 \%$ for electrons and lead.

\section{7. - Inelastic corrections.}

It is known that the deep inelastic contribution to the process under study is negligible $\left({ }^{5}\right)$, but there are other intermediate regions which are worth to consider. We study the region of small $x_{1}$ values not included in the quasielastic peak, for which the nuclear excitation energies $\nu \equiv-(P q)=-M q^{0}=$ $=M \omega$ are not linked to $x_{1}$.

In this region one can use the retarded dipole approximation, relating the 
hadronic structure functions to the photoabsorption cross-section

$$
W_{1}\left(x_{1}, v\right) \simeq \frac{v^{2}}{M^{2} x_{1}} W_{2}\left(x_{1}, v\right) \simeq \frac{v}{(2 \pi)^{2} \alpha} \sigma_{\gamma}(v)\left|F\left(x_{1}\right)\right|^{2} .
$$

The form factor $F\left(x_{1}\right)$ is needed to cut the contribution of large $x_{1}$ values, for which the unretarded approximation would not be appropriate. In our case, it suffices to take

$$
F\left(x_{1}\right)=\frac{a}{a+x_{1}}
$$

where $a$ is related to the nuclear radius $a=6\left\langle r^{2}\right\rangle^{-1}$. In the inelastic contribution, the two variables of the photon $x_{1}$ and $\omega$ are relevant, and it is important the comparison of their effective values against the new scale $a$.

For the muonic case, the restriction $x_{1} \leqslant a$ leads to consider the approximation $x_{1} \ll m^{2}$ as appropriate. This simplifies notably the leptonic tensor. Our aim is to write the cross-section of the process as a sum rule over the different energies $\omega$, with an explicit function $f(\omega, a)$ weighting the photon cross-section $\sigma_{\gamma}(\omega)$. After a lengthy calculation we get

$$
\sigma \simeq g(N, \lambda) \frac{3 \sigma_{0}}{(2 \pi)^{2} \alpha} E_{1} a \int \mathrm{d} \omega f(\omega, a) \sigma_{\gamma}(\omega),
$$

where the function $f(\omega, a)$ is plotted in fig. 6 for an incoming energy $E_{1}=$ $=100 \mathrm{GeV}$ and $a=2.4 \cdot 10^{-3}(\mathrm{GeV})^{2}$, as corresponds to the ${ }^{208} \mathrm{~Pb}$ target. The limits of $f(\omega, a)$ for $\left(\omega^{2} / a\right) \ll 1$ and $\left(\omega^{2} / a\right) \gg 1$ are also given in the figure, and they correspond to the analytic expressions

$$
\left\{\begin{array}{l}
f(\omega, a) \underset{\omega^{2} / a \ll 1}{\longrightarrow} f_{<}=\frac{\pi \sqrt{a}}{6 \omega}\left(\ln \left(2 E_{1} \sqrt{a} / m^{2}\right)-25 / 8\right) \\
f(\omega, a) \underset{\omega \omega^{2} / a \gg 1}{\longrightarrow} f_{>}= \\
=\frac{a}{3 \omega^{2}}\left(11 / 48-\frac{37}{12} \ln (2 \omega / \sqrt{a})+\ln \left(2 E_{1} \sqrt{a} / m^{2}\right)(\ln (2 \omega / \sqrt{a})-1 / 12)\right) .
\end{array}\right.
$$

As we see, for all the $\omega$ values one has the inequality $f_{<}>f(\omega, a)>f_{>}$, so $f_{<}$can be used to obtain an overestimate of this inelastic contribution to the total cross-section. The result is

$$
\sigma<g(N, \lambda) \frac{\sigma_{0} a^{\frac{3}{3}}}{8 \pi \alpha} E_{1}\left(\ln \left(2 E_{1} \sqrt{a} / m^{2}\right)-25 / 8\right) \sigma_{-1},
$$

where $\sigma_{-1}$ corresponds to the energy-weighted sum rule

$$
\sigma_{-1} \equiv \int \frac{\mathrm{d} \omega}{\omega} \sigma_{\gamma}(\omega)
$$




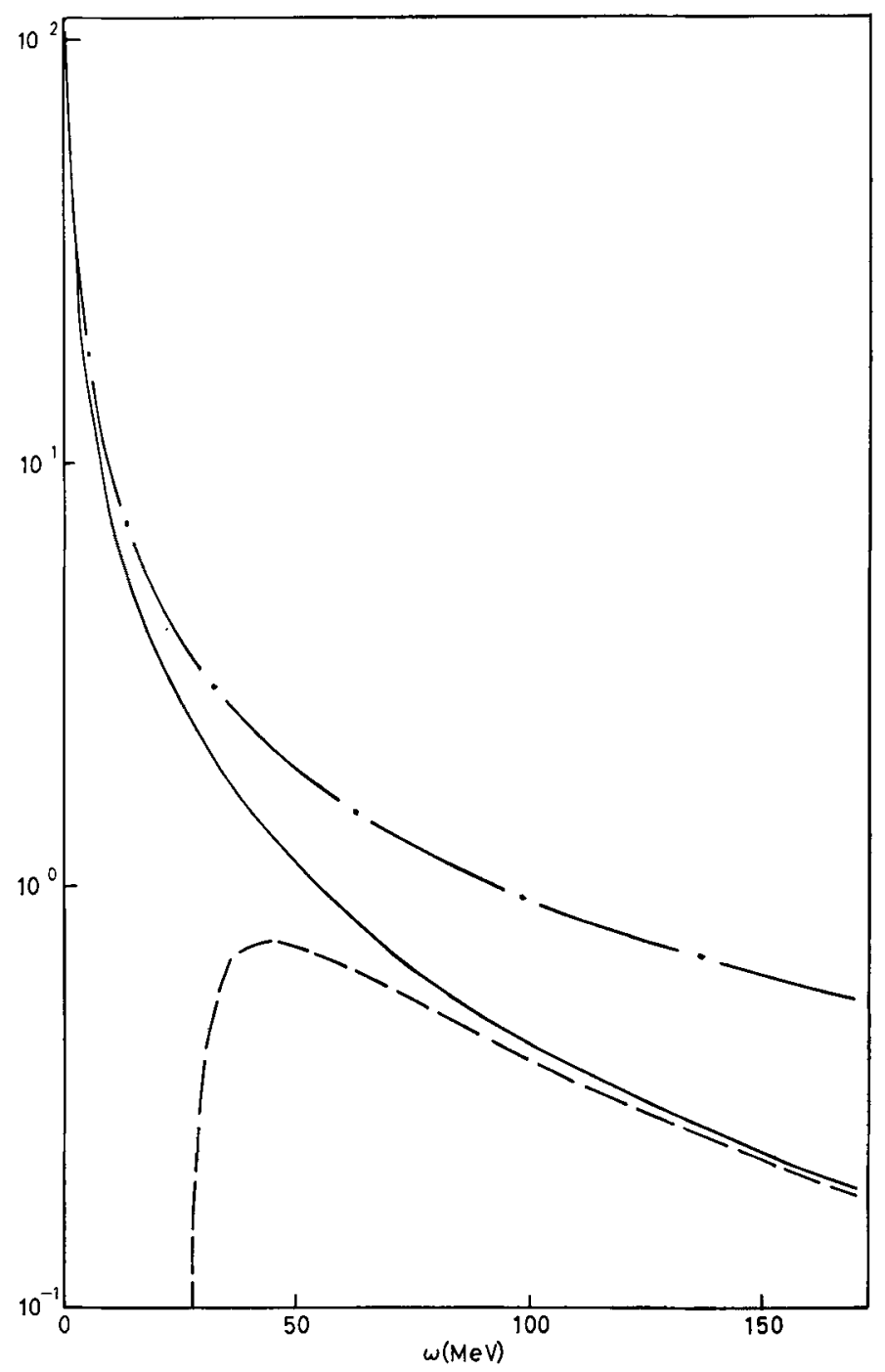

Fig. 6. - Values of $f(\omega, a)(-), f_{<}(-\cdot-\cdot-)$ and $f_{>}(---)$, as a function of $\omega$, for $E_{1}=100 \mathrm{GeV}$ and $a=2.4 \cdot 10^{-3}(\mathrm{GeV})^{2}$.

For heavy nuclei $(A \geqslant 100), \sigma_{-1}$ is well fitted $\left(^{(}\right)$by $\sigma_{-1}=(0.20 \pm 0.02) A^{\frac{4}{3}} \mathrm{mb}$, which can be used to evaluate expression (36). At the incoming energy $E_{1}=$ $=100 \mathrm{GeV}$, and for ${ }^{208} \mathrm{~Pb}$, we get $\sigma<2 \cdot 10^{-44} \mathrm{~g}(N, \lambda) \mathrm{cm}^{2}$, which is about four orders of magnitude smaller than the coherent contribution. So we conclude that the region of nuclear excitations at low $x_{1}$ is also negligible.

$\left({ }^{9}\right)$ O. Bohrgas : Proceedings of the International Conference on Theory and Applications of Moments Methods in Many-Fermion Systems, Iowa, 1979 (Plenum Press, New York, N. Y., 1980). 


\section{8. - Conclusion.}

We have considered the production of neutrino pairs by high-energy charged leptons. The main contribution results from the coherent nuclear Coulombic source. Corrections coming from the quasi-elastic peak are appreciable, whereas other inelastic nuclear excitations are negligible.

Allowing for an arbitrary polarization of the lepton beam, there are contributions to the process for both charged-current and neutral-current weak interactions. The dependence on the number of generations and on the helicity of the incoming lepton amounts to a global factor $g(N, \lambda)$ given by eq. (17) which reproduces $g\left(N,+\frac{1}{2}\right)=N$. Unless $N$ is very large, the charged-current contribution is dominant for left-handed helicity.

The energy spectrum of the outgoing charged lepton is peaked at low energies, with a maximum around six times its mass. There is, however, a tail of the distribution which produces a mean value of the energy somewhat higher and given in table II. In any case, we observe that the charged lepton loses most of its energy in the process of radiating the neutrino pair.

The angular distribution of the outgoing charged lepton is strongly peaked in the forward direction. Nevertheless, one obtains appreciable mean values of the dispersion angle as indicated in table II.

The results presented in this paper for the distributions of the outgoing charged lepton correspond to incoming energies up to $300 \mathrm{GeV}$, as the ones of muon beams at present facilities. In case that future facilities will provide lepton beams at higher energies, the corresponding distributions can be obtained from the authors.

\section{$* * *$}

This work has been supported in part by Comisión Asesora de Investigación Científica y Técnica, Spain. One of us (AP) acknowledges Caja de Ahorros de Valencia for a fellowship.

APPENDIX A

\section{Fierz transformation.}

We restrict ourselves to left-handed helicity and zero-mass fermions, so we can eliminate the $\gamma_{5}$ matrices in eq. (3) by using the fact that spinors are eigenvectors of the $\gamma_{5}$ operator, i.e.

$$
\gamma_{5} u\left(\lambda=-\frac{1}{2}\right)=u\left(\lambda=-\frac{1}{2}\right)
$$


We introduce the set of 16 Dirac matrices

$$
\Gamma^{i} \equiv\left(I, \gamma_{5}, \gamma^{\mu}, \gamma_{5} \gamma^{\mu}, \sigma^{\mu \nu}\right)
$$

and their corresponding inverses $\Gamma_{i} \equiv\left(\Gamma^{i}\right)^{-1}$. Inserting the identity $\left.{ }^{10}\right)$

$$
\delta_{a, \vec{a}} \delta_{b, \bar{b}}=\frac{1}{4}\left(\Gamma_{i}\right)_{\bar{b} \bar{a}}\left(\Gamma^{i}\right)_{a, b}
$$

in the product of weak currents that appears in the charged amplitudes, we can write

$$
\left\{\begin{array}{l}
N^{3 \sigma} L_{\sigma \mu}^{3} \simeq\left(\bar{u}\left(p_{2}\right) \Gamma^{i} T_{\sigma \mu} u\left(p_{1}\right)\right)\left(\bar{u}\left(p_{4}\right) \Gamma_{i} \gamma^{\sigma} v\left(p_{3}\right)\right) \\
N^{4 \sigma} L_{\mu \sigma}^{4} \simeq\left(\bar{u}\left(p_{2}\right) \tilde{T}_{\mu \sigma} \Gamma_{i} u\left(p_{1}\right)\right)\left(\bar{u}\left(p_{4}\right) \gamma^{\sigma} \Gamma^{i} v\left(p_{3}\right)\right)
\end{array}\right.
$$

where

$$
T_{\sigma \mu} \equiv \gamma_{\sigma}\left(p_{1}+q\right) \gamma_{\mu}, \quad \tilde{T}_{\mu \sigma} \equiv \gamma_{\mu}\left(p_{2}-q\right) \gamma_{\sigma}
$$

For zero-mass particles, the fermionic current preserves or changes the helicity according to the odd or even number of gamma matrices it contains; so, only the even operators, $I, \gamma_{5}$ and $\sigma^{\mu \nu}$, will contribute to eqs. (A.4). The $\gamma_{5}$ matrices can be eliminated by using again identity (A.1). For the terms containing the $\sigma_{\alpha \beta}$ operator we make the expansion

$$
X=\frac{1}{4} \operatorname{Tr}\left(X \Gamma_{i}\right) \Gamma^{i}
$$

obtaining

$$
\left\{\begin{aligned}
N^{3 \sigma} L_{\sigma \mu}^{3} & \simeq 2\left(\bar{u}\left(p_{2}\right) T_{\sigma \mu} u\left(p_{1}\right)\right)\left(\bar{u}\left(p_{4}\right) \gamma^{\sigma} v\left(p_{3}\right)\right)+ \\
& +\frac{1}{32} \operatorname{Tr}\left(\sigma_{\alpha \beta} T_{\sigma \mu} \Gamma_{i}\right) \operatorname{Tr}\left(\sigma^{\alpha \beta} \gamma^{\sigma} \Gamma_{j}\right)\left(\bar{u}\left(p_{2}\right) \Gamma^{i} u\left(p_{1}\right)\right)\left(\bar{u}\left(p_{4}\right) \Gamma^{j} v\left(p_{3}\right)\right) \\
N^{4 \sigma} L_{\mu \sigma}^{4} & \simeq 2\left(\bar{u}\left(p_{2}\right) \tilde{T}_{\mu \sigma} u\left(p_{1}\right)\right)\left(\bar{u}\left(p_{4}\right) \gamma^{\sigma} v\left(p_{3}\right)\right)+ \\
& +\frac{1}{32} \operatorname{Tr}\left(\widetilde{T}_{\mu \sigma} \sigma_{\alpha \beta} \Gamma_{i}\right) \operatorname{Tr}\left(\gamma^{\sigma} \sigma^{\alpha \beta} \Gamma_{j}\right)\left(\bar{u}\left(p_{2}\right) \Gamma^{i} u\left(p_{1}\right)\right)\left(\bar{u}\left(p_{4}\right) \Gamma^{j} v\left(p_{3}\right)\right) .
\end{aligned}\right.
$$

The helicity argument indicates now that the only contributions come from the odd operators $\gamma^{\mu}$ and $\gamma_{5} \gamma^{\mu}$. Eliminating the $\gamma_{5}$ matrices, we can write

$$
\left\{\begin{array}{l}
N^{3 \sigma} L_{\sigma \mu}^{3} \simeq\left(\bar{u}\left(p_{4}\right) \gamma^{\sigma} v\left(p_{3}\right)\right)\left(\left(2\left(\bar{u}\left(p_{2}\right) T_{\sigma \mu} u\left(p_{1}\right)\right)+\frac{1}{32} s_{\mu}^{\varrho \sigma}\left(\bar{u}\left(p_{2}\right) \gamma_{\varrho} u\left(p_{1}\right)\right)\right)\right. \\
N^{4 \sigma} L_{\mu \sigma}^{4} \simeq\left(\bar{u}\left(p_{4}\right) \gamma^{\sigma} v\left(p_{3}\right)\right)\left(2\left(\bar{u}\left(p_{2}\right) \tilde{T}_{\mu \sigma} u\left(p_{1}\right)\right)+\frac{1}{32} t_{\mu}^{\varrho \sigma}\left(\bar{u}\left(p_{2}\right) \gamma_{\varrho} u\left(p_{1}\right)\right)\right)
\end{array}\right.
$$

where $s_{\mu}^{e \sigma}$ and $t_{\mu}^{0 \sigma}$ are combinations of gamma traces. We get after some algebra

$$
s_{\mu}^{\varrho \sigma}=48 \operatorname{Tr}\left(T^{\sigma}{ }_{\mu} \gamma^{\varrho}\left(\gamma_{5}-1\right)\right), \quad t_{\mu}^{\varrho \sigma}=48 \operatorname{Tr}\left(\widetilde{T}_{\mu}^{\sigma} \gamma^{\varrho}\left(\gamma_{5}-1\right)\right) .
$$

(10) C. ITzYkson and J. B. Zuber: Quantum Field Theory (McGraw-Hill, New York, N. Y., 1980), p. 161. 
To compare with the neutral-current amplitudes, we make the expansion (A.6) in $L_{\sigma \mu}^{1}$ and $L_{\mu \sigma}^{2}$ and uso again the helicity argument, obtaining

$$
\left\{\begin{aligned}
\frac{L_{\sigma \mu}^{1}}{v-1} & \simeq \frac{1}{4} \operatorname{Tr}\left(T_{\sigma \mu} \Gamma_{i}\right)\left(\bar{u}\left(p_{2}\right) \Gamma^{i} u\left(p_{1}\right)\right) \\
& \simeq \frac{1}{4} \operatorname{Tr}\left(T_{\sigma \mu} \gamma_{\varrho}\left(1-\gamma_{5}\right)\right)\left(\bar{u}\left(p_{2}\right) \gamma^{\varrho} u\left(p_{1}\right)\right), \\
\frac{L_{\mu \sigma}^{2}}{v-1} & \simeq \frac{1}{4} \operatorname{Tr}\left(\tilde{T}_{\mu \sigma} \Gamma_{i}\right)\left(\bar{u}\left(p_{2}\right) \Gamma^{i} u\left(p_{1}\right)\right) \simeq \\
& \simeq \frac{1}{4} \operatorname{Tr}\left(\widetilde{T}_{\mu \sigma} \gamma_{\varrho}\left(1-\gamma_{5}\right)\right)\left(\bar{u}\left(p_{2}\right) \gamma^{\varrho} u\left(p_{1}\right)\right),
\end{aligned}\right.
$$

Comparing now eqs. (A.8), (A.9) and (A.10), wo have the desired result (4).

\section{APPENDIX B}

\section{Phase space for the energy distribution.}

The squared invariant mass of the $v \bar{\nu}$ pair, $k^{2}$, is related to the variable $z$ through the equation

$$
z=x_{2}-\frac{1}{2} x_{1}+m^{2}-\frac{1}{2} k^{2}
$$

so, the kinematical limits on $k^{2}$ impose $z_{1} \leqslant z \leqslant z_{3}$, where

$$
z_{1}=x_{2}-\frac{1}{2} x_{1}+m^{2}-\frac{1}{2}\left(E_{1}+q^{0}-E_{2}\right)^{2}, \quad z_{3}=x_{2}-\frac{1}{2} x_{1}+m^{2}
$$

Another set of boundaries come from the $-1 \leqslant \cos \theta_{2} \leqslant 1$ condition, which imposes $z_{2} \leqslant z \leqslant z_{4}$, where

$$
z_{2}=E_{2}\left(E_{1}+q^{0}\right)-\left|\boldsymbol{p}_{2}\right| \sqrt{W}, \quad z_{4}=E_{2}\left(E_{1}+q^{0}\right)+\left|\boldsymbol{p}_{2}\right| \sqrt{W}
$$

One easily checks that $z_{2} \geqslant z_{1}$, so $\underline{z}=z_{2}$. To obtain the upper $z$ limit is not trivial at all, because there are several cuts among $z_{3}, z_{4}$ and $z_{2}$. These boundaries restrict also the allowed $x_{2}$ values, because of the existence of regions where $z_{2}>z_{3}$, so the $x_{2}$ limits do not always take the values given in ref. $\left({ }^{2}\right)$. We obtain

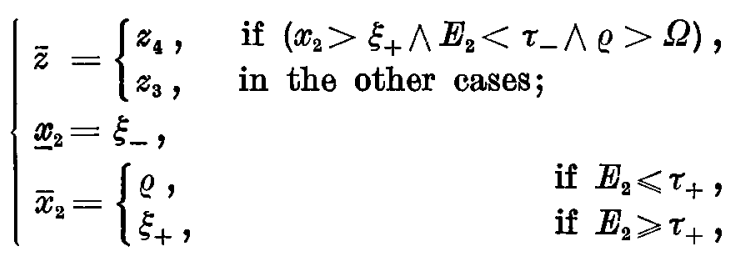


where $\xi_{ \pm}$are the $x_{2}$-values for which $z_{3}$ cuts to $z_{2}$ or $z_{4}$

$$
\xi_{ \pm} \equiv \frac{1}{2} x_{1}+\left(E_{1}+q^{0}-E_{2}\right)\left(E_{2} \pm\left|\boldsymbol{p}_{2}\right|\right) .
$$

$\varrho$ is the upper limit of $x_{2}$ obtained from the $\cos \theta_{q} \geqslant-1$ condition

$$
\varrho \equiv\left|\boldsymbol{p}_{1}\right|\left(x_{1}+q^{02}\right)^{\frac{1}{2}}+E_{1} q^{0}
$$

$\tau_{ \pm}$are the outgoing energies at which the cut $\varrho=\xi_{ \pm}$occurs

$$
\tau_{ \pm} \equiv \frac{\left(E_{1}+q^{0}\right)\left(\varrho-\frac{1}{2} x_{1}+m^{2}\right) \pm\left(\varrho-\frac{1}{2} x_{1}\right)\left(\left(E_{1}+q^{0}\right)^{2}-2 \varrho+x_{1}-m^{2}\right)^{\frac{1}{2}}}{2 \varrho-x_{1}+m^{2}}
$$

and

$$
\Omega \equiv \frac{1}{2} x_{1}+m\left(E_{1}+q^{0}-m\right)
$$

The relation between $\Omega$ and $\varrho$ regulates whether $\xi_{+}$or $\xi_{-}$is the one which cuts $\varrho$ in $E_{2}=\tau_{-}$.

In the high-energy limit $\left(E_{1} \gg m, x_{1}^{\frac{1}{1}}\right), \tau_{ \pm}$can be approximated by

$$
\tau_{+} \simeq E_{1}-\frac{1}{2} x_{1}^{\frac{1}{1}}, \quad \tau_{-} \simeq \frac{1}{2} x_{1}^{\frac{1}{4}}+\frac{m^{2}}{2 x_{1}^{\frac{1}{4}}}\left(1+\frac{m^{2}-x_{1}}{2 E_{1} x_{1}^{\frac{1}{4}}}\right)^{-1} .
$$

For $\varrho<\Omega$ the $E_{2}<\tau_{-}$region is not allowed, because $\xi_{-}>\varrho$. This imposes a lower limit over $x_{1}$. Using (B.9), we obtain

$$
\underline{x_{1}^{1}} \simeq \frac{E_{1} E_{2}}{E_{1}+E_{2}}\left(1-\left(1-m^{2} \frac{E_{1}^{2}-E_{2}^{2}}{E_{1}^{2} E_{2}^{2}}\right)^{\frac{1}{2}}\right)
$$

APPENDIX C

\section{Phase space for the angular distribution.}

Similarly to the energy phase-space distribution, we obtain two sets of boundaries over the variable $y$ from the $-1 \leqslant \cos \theta_{q} \leqslant 1$ condition and the $k^{2}$ kinematical limits. However, the situation is now simpler and one obtains

$$
\underline{y}=x_{5}+\frac{1}{2} x_{1}-m^{2}, \quad \bar{y}=q^{0}\left(E_{1}-E_{2}\right)+|\boldsymbol{q}| H^{\frac{1}{2}}
$$

The allowed phase-space region must satisfy $\underline{y} \leqslant \bar{y}$. This restricts the possible $x_{5}$ values to $x_{5}^{-} \leqslant x_{5} \leqslant x_{5}^{+}$, where

$$
x_{5}^{ \pm} \equiv \frac{1}{2} x_{1}+m^{2}+\left(q^{0} \pm|\boldsymbol{q}|\right)\left(E_{1}-E_{2}+q^{0}\right)
$$


As $x_{5}$ depends on $E_{2}$ and $\theta_{2}$, the $x_{5}$ restrictions impose boundaries over these variables. A lower limit over $x_{1}$ is also obtained from the $x_{1}$ dependence of $x_{5}^{ \pm}$. One obtains

$$
\left\{\begin{array}{lr}
\bar{E}_{2}=\left\{\begin{array}{lr}
\zeta, & \text { if } \cos \theta_{2} \leqslant 1-x_{1} / 2\left|\boldsymbol{p}_{1}\right|^{2}, \\
\phi, & \text { if } \cos \theta_{2} \geqslant 1-x_{1} / 2\left|\boldsymbol{p}_{1}\right|^{2},
\end{array}\right. \\
\underline{E}_{2}=m, & \text { if } \cos \theta_{2} \leqslant 0, \\
\underline{x}_{1} \simeq\left\{\begin{array}{lr}
m^{2}, & \text { if } \cos \theta_{2} \geqslant 0, \\
m^{2}\left(1-\cos ^{2} \theta_{2}\right), &
\end{array}\right.
\end{array}\right.
$$

where

$$
\left\{\begin{array}{l}
\zeta \equiv \frac{\left(E_{1}+x_{1}^{\frac{1}{1}}\right)\left(E_{1} x_{1}^{\frac{1}{1}}+\frac{1}{2} x_{1}+m^{2}\right)+\left|\boldsymbol{p}_{1}\right| \cos \theta_{2}\left(x_{1}\left(E_{1}+\frac{1}{2} x_{1}^{\frac{1}{1}}\right)^{2}-m^{2}\left|\boldsymbol{p}_{1}\right|^{2}\left(1-\cos ^{2} \theta_{2}\right)\right)^{\frac{1}{2}}}{\left(E_{1}+x_{1}^{\frac{1}{2}}\right)^{2}-\left|\boldsymbol{p}_{1}\right|^{2} \cos ^{2} \theta_{2}} \\
\phi \equiv \frac{\left(E_{1}-x_{1}^{\frac{1}{2}}\right)\left(E_{1} x_{1}^{\frac{1}{2}}-\frac{1}{2} x_{1}-m^{2}\right)-\left|\boldsymbol{p}_{1}\right| \cos \theta_{2}\left(x_{1}\left(E_{1}-\frac{1}{2} x_{1}^{\frac{1}{2}}\right)^{2}-m^{2}\left|\boldsymbol{p}_{1}\right|^{2}\left(1-\cos ^{2} \theta_{2}\right)\right)^{\frac{1}{2}}}{\left|\boldsymbol{p}_{1}\right|^{2}} \cos ^{2} \theta_{2}-\left(E_{1}-x_{1}^{\frac{1}{2}}\right)^{2}
\end{array}\right.
$$

\section{RIASSUNTO(*)}

Si calcola la sezione d'urto per la produzione di coppie $v-\bar{\nu}$ per leptoni ad alta energia nel campo nucleare di Coulomb nella teoria standard, tenendo conto della polarizzazione arbitraria nel fascio leptonico incidente. Si studiano le distribuzioni differenziali del leptone carico uscente, mostrando che il leptone diffuso forma un picco ad alta energia ed emerge per angoli di un'ampiezza notevole. Si discutono anche i contributi incoerenti alla sezione d'urto.

(*) Traduzione a cura della Redazione.

Лепто-рождение нейтринных пар в ядерном кулоновском поле.

Резюме (*). - Вычисляется поперечное сечение рождения у-̄̄ пар лептонами высоких энергий в ядерном кулоновском поле в рамках стандартной теории, предполагая произвольную поляризацию падающего пучка лептонов. Исследуются дифференциальные распределения вылетающих заряженных лептонов. Показывается, что существуют пики заряженных лептонов при низких энергиях и заметных углах. Также обсуждаются некогерентные вклады в поперечное сечение.

\section{(") Переведено редакчией.}

